\title{
A CMOS current-mode operational amplifier
}

\author{
Kaulberg, Thomas
}

Published in:

I E E E Journal of Solid State Circuits

Link to article, DOI:

10.1109/4.222187

Publication date:

1993

Document Version

Publisher's PDF, also known as Version of record

Link back to DTU Orbit

Citation (APA):

Kaulberg, T. (1993). A CMOS current-mode operational amplifier. I E E E Journal of Solid State Circuits, 28(7), 849-852. https://doi.org/10.1109/4.222187

\section{General rights}

Copyright and moral rights for the publications made accessible in the public portal are retained by the authors and/or other copyright owners and it is a condition of accessing publications that users recognise and abide by the legal requirements associated with these rights.

- Users may download and print one copy of any publication from the public portal for the purpose of private study or research.

- You may not further distribute the material or use it for any profit-making activity or commercial gain

- You may freely distribute the URL identifying the publication in the public portal

If you believe that this document breaches copyright please contact us providing details, and we will remove access to the work immediately and investigate your claim. 


\title{
A CMOS Current-Mode Operational Amplifier
}

\author{
Thomas Kaulberg
}

\begin{abstract}
A fully differential input differential output currentmode operational amplifier (COA) is described. The amplifier utilizes three second-generation current conveyors (CCII's) as the basic building blocks. It can be configured to provide either a constant gain-bandwidth product in a fully balanced current-mode feedback amplifier or a constant bandwidth in a transimpedance feedback amplifier. The amplifier is found to have a gain-bandwidth product of $3 \mathrm{MHz}$, an offset current of $0.8 \mu \mathrm{A}$ (signal range $\pm 700 \mu \mathrm{A}$ ), and a (theoretically) unlimited slew rate. The amplifier is realized in a standard CMOS $2.4-\mu \mathrm{m}$ process.
\end{abstract}

\section{INTRODUCTION}

$\mathbf{T}$ HE traditional operational amplifier and the transimpedance operational amplifier (also known as currentfeedback operational amplifier) are unquestionably two of the most flexible and widely used analog integrated circuits of today. However, as the standard supply voltages for digital circuitry are decreasing significantly, so are the signal ranges for the analog components (especially in mixed analog- and digital-mode systems). An attempt to overcome this problem can be simply to change the signal representation from a voltage to a current. In this way the signal range is no longer directly restricted by the supply voltage but also dependent on the impedance level chosen by the designer.

In the process of designing current-mode circuits it can be advantageous to follow the Theory of Adjoint Networks [1], [2]. This theory is a conversion strategy which, when applied to a given voltage-mode circuit, leads to a current-mode circuit with the same transfer function. This paper describes an attempt to obtain a useful structure for a current-mode op amp by applying the Theory of Adjoint Networks to the traditional op amp and to the transimpedance op amp on a subcircuit level. Most current amplifiers described in the literature have only limited gain [3], [4]. However, the structure described in the present paper is suitable for true COA operations, i.e. a current gain in the order of $80 \mathrm{~dB}$.

\section{ADJOINT NETWORK CONVERSION}

The second-generation current conveyor is a versatile building block for a variety of applications [5]. It offers both a current-following and a voltage-following function and is useful in expressing both current-mode and voltage-mode circuits at a subcircuit level. A symbol and a simple CMOS implementation of a CCII+ is shown in Fig. 1. The transfer

Manuscript received December 11, 1992; revised March 15, 1993. This work was performed as parts of M.Sc. and Ph.D. study under the supervision of Prof. E. Bruun.

The authors are with the Electronics Institute, Technical University of Denmark, DK-2800 Lyngby, Denmark.

IEEE Log Number 9209013.

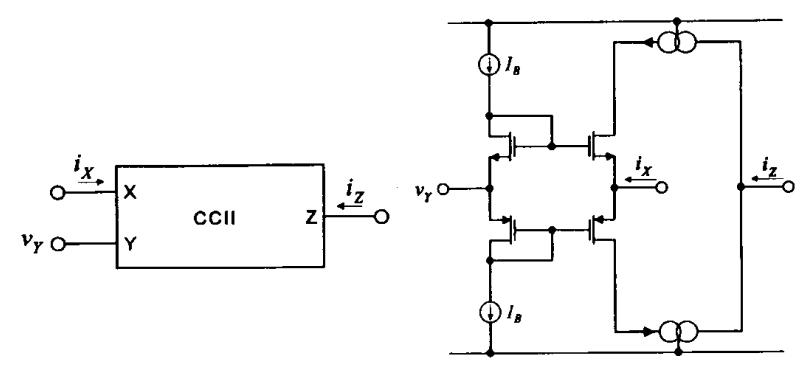

Fig. 1. The second-generation current conveyor.

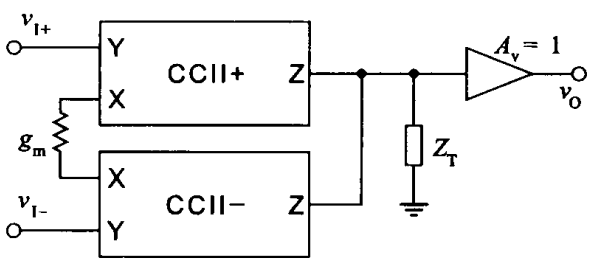

Fig. 2. Voltage-mode op amp (VOA).

matrix is given by the following equation:

$$
\left[\begin{array}{c}
i_{Y} \\
v_{X} \\
i_{Z}
\end{array}\right]=\left[\begin{array}{ccc}
0 & 0 & 0 \\
1 & 0 & 0 \\
0 & \pm 1 & 0
\end{array}\right]\left[\begin{array}{l}
v_{Y} \\
i_{X} \\
v_{Z}
\end{array}\right]
$$

The CCII- contains both a positive voltage-following action and a positive current-following action and according to the Adjoint Networks Theory it is its own interreciprocal circuit [2]. This means that the adjoint network of a CCIIis a CCII-. The CCII+ exhibits a positive voltage-following action and a negative current-following action. The adjoint network of this circuit is a circuit with a negative voltage follower and a positive current follower. Obviously, it is more complicated to generate a precise negative voltage follower than a positive one. Therefore we shall try to avoid this type of component in our generation of current-mode circuits.

Fig. 2 shows a traditional voltage-mode op amp (VOA) expressed in terms of current conveyors. The differential input voltage is transferred via a transconductance, $g_{m}$, to a differential current which is sourced into an impedance, $Z_{T}$, and the voltage across this impedance is buffered to the output of the VOA. The transimpedance $Z_{T}$ is the parallel connection of a large resistance and the compensation capacitor $C_{C}$. This gives the well-known transfer function with one dominant pole, a large dc gain, and a constant gain-bandwidth product.

By applying the Theory of Adjoint Networks to this circuit we achieve a current operational amplifier (COA) as shown in Fig. 3. With the chosen sign convention, all currents are considered positive in the direction of the arrow. The 


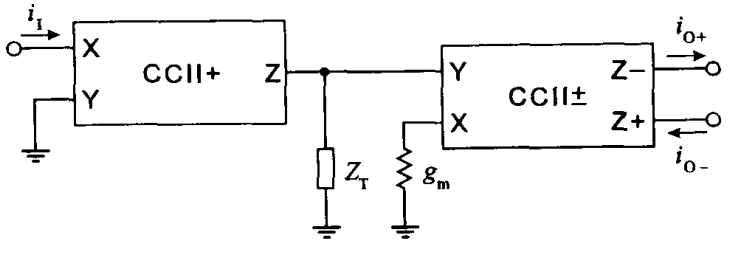

Fig. 3. Current-mode op amp (COA).

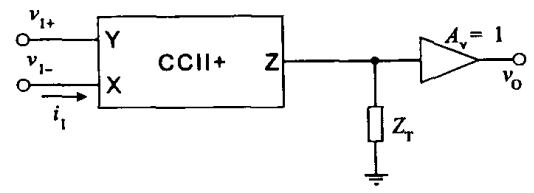

Fig. 4. Voltage-mode transimpedance amplifier.

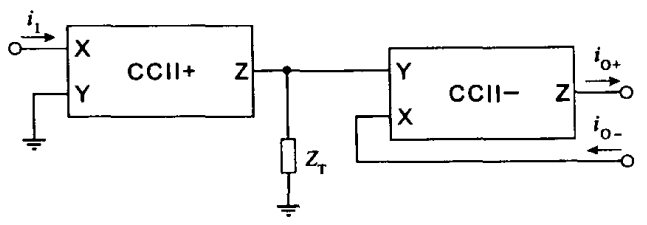

Fig. 5. Current-mode transimpedance amplifier.

output buffer of the VOA turns into a current buffer. The current buffer would normally be realized as a CCII- with $V_{Y}=0$. In this case it is realized as a CCII+ with $V_{Y}=0$. The change of sign is compensated in the following stage. The two CCII's that form the differential transconductance stage $\left(I_{o u t}=g_{m} V_{d, i n}\right)$ in the VOA turn into a differential transconductance output CCII $\pm\left(I_{d, o u t}=g_{m} V_{i n}\right)$. Passive elements like resistors and capacitors remain unchanged. The current gain, $I_{d, o} / I_{i}$, of this circuit is exactly the same as the voltage gain, $V_{o} / V_{d, i}$, of the VOA [6].

A simplified diagram of a transimpedance operational amplifier [7] is shown in Fig. 4. The differential input stage of this amplifier contains both a high-impedance voltage input node and a low-impedance current input node. The current from this node is conveyed to the transimpedance $Z_{T}$ and the voltage across $Z_{T}$ is buffered to the output. In a feedback configuration this amplifier provides a nearly constant bandwidth, independent of the closed-loop gain [7].

When converting to current mode, the CCII + , which forms the input stage of the voltage-mode transimpedance amplifier, is transformed into a CCII- and an additional inverting voltage buffer. The output buffer is transformed into a CCIIwith a grounded $Y$ input. By rearranging this circuit slightly we arrive at the circuit shown in Fig. 5. This circuit is called the current-mode transimpedance amplifier [8].

\section{Current-Mode Operational Amplifier (COA)}

When comparing Figs. 3 and 5 we find that the only difference between the two amplifiers is the connection of the low-impedance $X$ input of the output CCII-. By bringing this terminal to an external pin we obtain a highly flexible

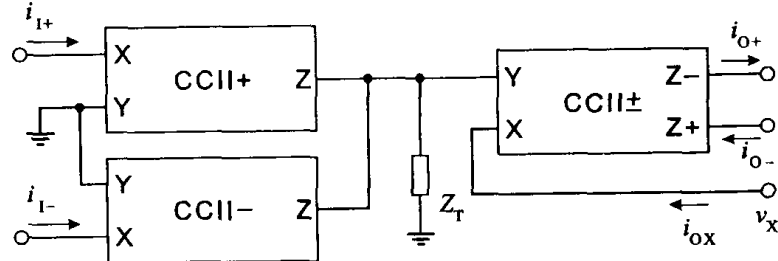

Fig. 6. Current-mode operational amplifier.

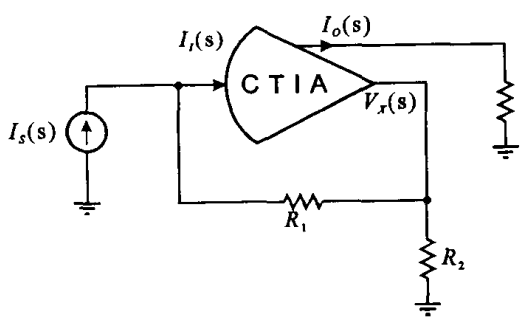

Fig. 7. Current amplifier.

current-mode operational amplifier. In Fig. 6 such an amplifier is shown. Another option is added to this figure. The current input is made differential. This makes the amplifier interreciprocal to a fully balanced VOA. To be interreciprocal to a single-output VOA, the $I_{i-}$ terminal should be left open.

The connection of the $V_{\mathrm{X}}$ terminal now determines the operational mode of the amplifier. When $V_{X}$ is connected to ground, the amplifier is interreciprocal to the traditional operational amplifier (VOA). We call this configuration a current operational amplifier.

From Fig. 3 the transfer function can easily be derived as shown below:

$$
\begin{gathered}
H_{\mathrm{COA}}(\mathrm{s})=\frac{I_{o}(\mathrm{~s})}{I_{i}(\mathrm{~s})}=\frac{g_{m} R_{T}}{1+R_{T} C_{C}} \\
\Downarrow A_{\mathrm{DC}, \mathrm{COA}}=g_{m} R_{T}, \quad \mathrm{~GB}_{\mathrm{COA}}=\frac{g_{m}}{C_{C}} .
\end{gathered}
$$

This amplifier provides a large $\mathrm{dc}$ gain, a constant gain-bandwidth product and a "one dominant pole" transfer function similar to the properties of the VOA. A $45^{\circ}$ phase margin is ensured by making GB $<2 \pi f_{2}$. GB is the gain-bandwidth product and $f_{2}$ is the second pole of the amplifier (typically determined by the current mirror pole $\simeq f_{T} / 2$.

When the $V_{X}$ terminal is used as a feedback terminal, the amplifier is configured as a current-mode transimpedance amplifier as shown in Fig. 5. Fig. 7 shows how a simple current amplifier with feedback can be realized with this component. The closed-loop gain for $R_{T} \gg R_{1}$ is

$$
\begin{aligned}
A_{i}(\mathrm{~s})=\frac{I_{o}(\mathrm{~s})}{I_{i}(\mathrm{~s})} & =\left(1+\frac{R_{1}}{R_{2}}\right) \frac{1}{1+R_{1} C_{C}} \\
\mathrm{BW} & =\frac{1}{R_{1} C_{C}} .
\end{aligned}
$$

Equation (3) shows how this amplifier exhibits a constant bandwidth property similar to that of a traditional voltagemode transimpedance operational amplifier. The stability criterion (phase margin $>45^{\circ}$ ) for this amplifier is $\mathrm{BW}<2 \pi f_{2}$. 


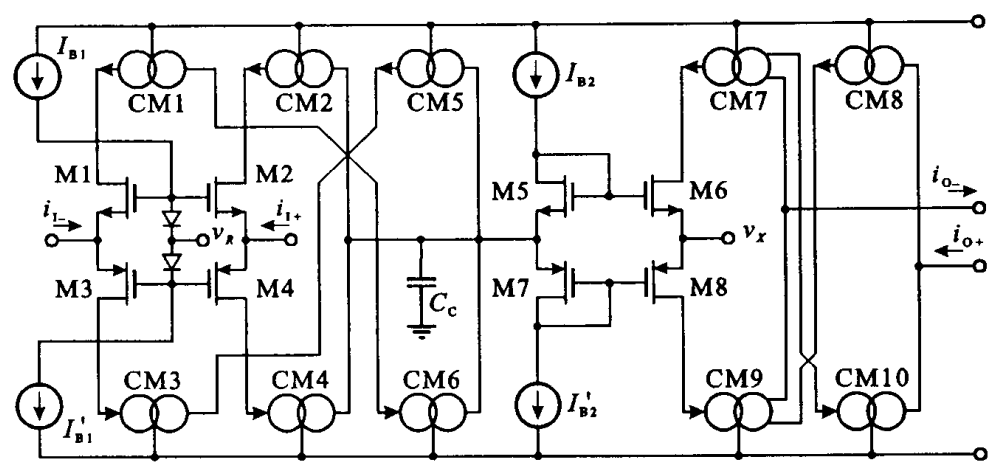

Fig. 8. Current-mode operational amplifier.

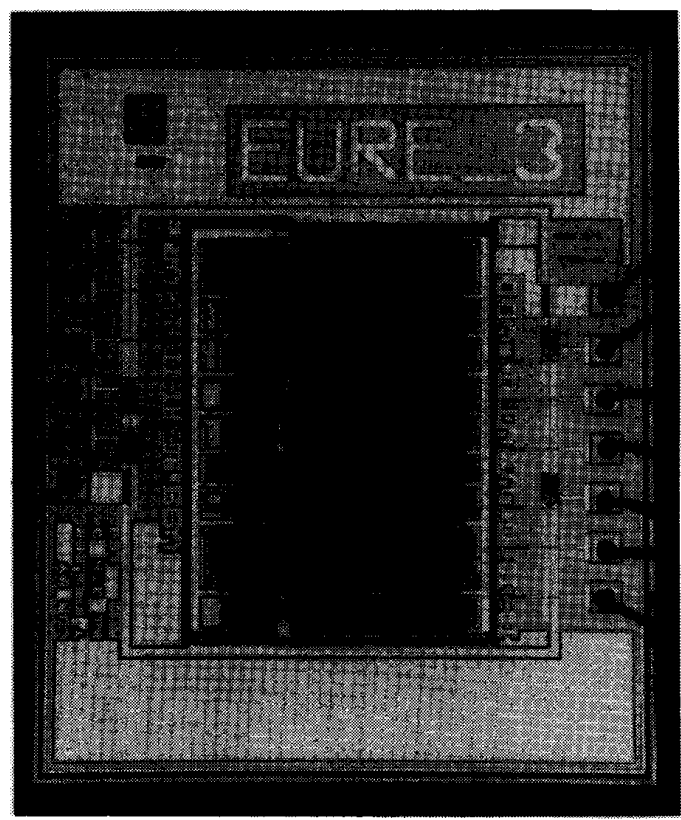

Fig. 9. Chip photo.

Both of the two amplifier configurations presented can replace their interreciprocal voltage-mode counterparts in any voltage-mode circuit due to the Theory of Adjoint Networks.

The two amplifier configurations do not suffer from slewrate limitation like most of the traditional VOA implementations do. The reason for this is the fact that the current available for charging and discharging $C_{C}$ is not limited to a quiescent value (bias current source) but only limited by the current signal range of the input current conveyors.

\section{IMPLEMENTATION}

The current-mode operational amplifier has been realized as shown in the diagram of Fig. 8. The circuit is characterized by (4) and (5):

$$
r_{x}=\frac{1}{g_{\mathrm{m} 6}+g_{\mathrm{mbs} 6}+g_{\mathrm{m} 8}+g_{\mathrm{mbs} 8}}
$$

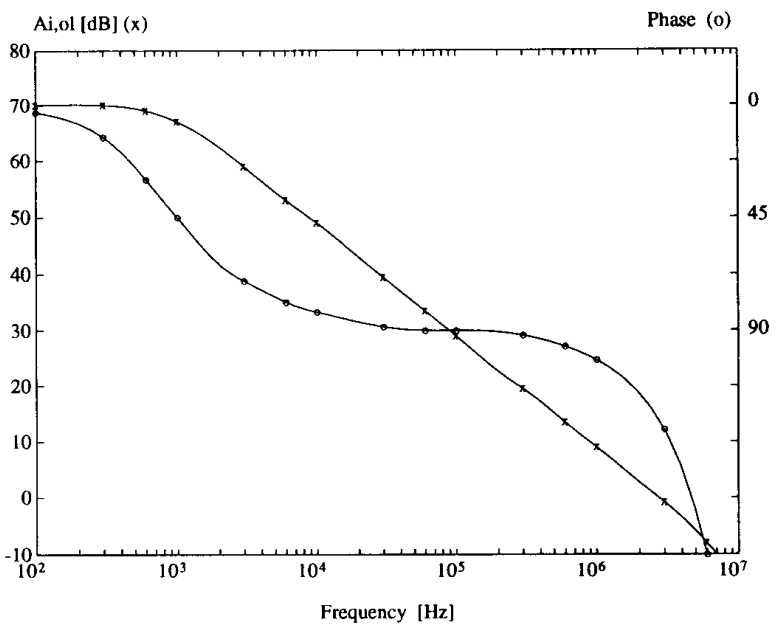

Fig. 10. Open-loop measurements of COA.

$$
R_{T}=\frac{1}{g_{\mathrm{ocm} 2}+g_{\mathrm{ocm} 4}+g_{\mathrm{ocm} 5}+g_{\mathrm{ocm} 6}+g_{\mathrm{oib} 2}+g_{\mathrm{oib} 2^{\prime}}}
$$

where $g_{\mathrm{mbs} n}$ is the bulk transconductance and $g_{\mathrm{o} n}$ represents the output conductance of the current mirrors and current sources. The input current offset errors are strongly dependent on the accuracy of the current mirrors [6] and therefore all current mirrors have been realized as cascoded current mirrors. Each transistor in the amplifier needs to match one or two other transistors, and in order to improve the matching large transistor dimensions $\left(L>L_{\min }\right)$ and a finger-structure common centroid layout technique have been used for all transistors.

\section{EXPERIMENTAL RESULTS}

The COA has been implemented in a $2.4-\mu \mathrm{m}$ industry standard CMOS process. A chip photo is shown in Fig. 9. The COA was found to have an open-loop current gain as shown in Fig. 10. It is seen that the dc gain is approximately $72 \mathrm{~dB}$ and the $\mathrm{GB}$ is $3 \mathrm{MHz}$ at a phase margin of $60^{\circ}$. The amplifier was optimized to achieve low offset current. This was measured to be $0.8 \mu \mathrm{A}$ compared to a signal range of $\pm 700 \mu \mathrm{A}$. 
$\mathrm{Ai}[\mathrm{dB}]$

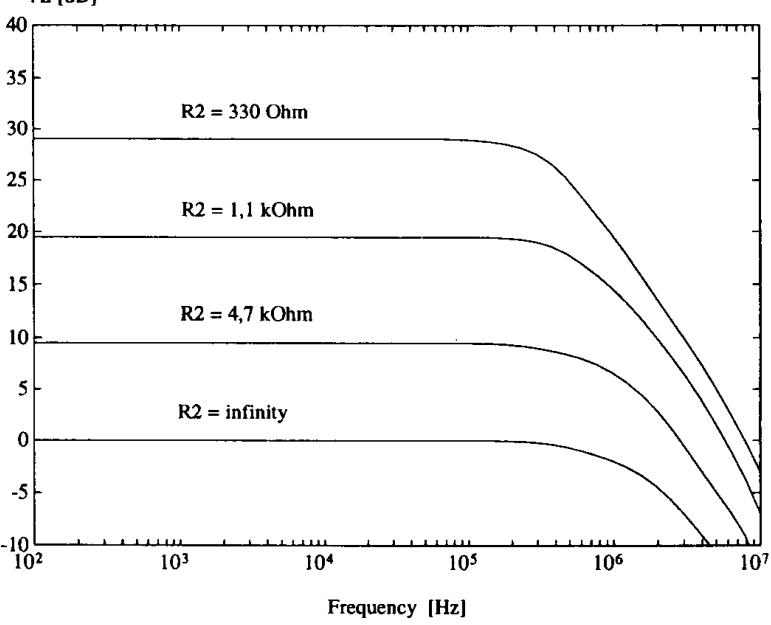

Fig. 11. Current gain of CTIA.

In Fig. 11 measured results from the feedback configuration of Fig. 7 are shown. The feedback resistor $R_{1}$ is $10 \mathrm{k} \Omega$ and different values of $R_{2}$ ranging from $330 \Omega$ to infinite have been used. The measurements confirm the expected constant bandwidth characteristics of the amplifier. $C_{C}$ was set to about $60 \mathrm{pF}$ in the VOA mode and about $2 \mathrm{pF}$ in the CTIA mode. The slew rate was found to be limited solely by the bandwidth of the $\mathrm{COA}$

\section{CONCLUSION}

A CMOS current-mode operational amplifier has been analyzed and experimental results have been presented. Due to a flexible interconnection of three second-generation current conveyors, a versatile current-mode building block has been achieved. The current-mode operational amplifier is found to have current processing properties equivalent to the voltage processing properties of a traditional balanced voltagemode operational amplifier and a transimpedance operational amplifier.

\section{REFERENCES}

[1] G. W. Roberts, "All current-mode frequency selective circuits," Electron. Lett., vol. 25, pp. 759-761, 1989.

[2] G. W. Roberts and A. S. Sedra, "Adjoint networks revisited," in Proc ISCAS, vol. 1, 1989, pp. 540-544.

[3] C. Toumazou, F. J. Lidgey, and D. G. Haigh, Analogue IC Design: The Current-Mode Approach. London: Peter Peregrinus, 1990, ch. 4.

[4] A. Fabre and P. Rochegude, "Current processing circuits with translinear operational current amplifiers," Int.J. Electron., vol. 63, pp. 9-28, 1987.

[5] A. S. Sedra and K. C. Smith, "A second-generation current conveyor and it's applications," IEEE Trans. Circuit Theory, vol. CT-17, pp. 132-134, 1970 .

[6] E. Bruun, "A differential-input differential-output current-mode operational amplifier," Int. J. Electron., vol. 71, pp. 1047-1056, 1991.

[7] D. Nelson and S. Evans, "A new approach to op amp design," Comlinear Corp., Appl. Note 300-301, Mar. 1985.

[8] E. Bruun, "A constant-bandwidth current-mode operational amplifier," Electron. Lett., vol. 27, pp. 1673-1674, 1991. 